\title{
Attitudes, Awareness, and Barriers Regarding Evidence-Based Surgery Among Surgeons and Surgical Nurses
}

\author{
Anouk M. Knops · Hester Vermeulen • \\ Dink A. Legemate · Dirk T. Ubbink
}

Published online: 2 May 2009

(C) The Author(s) 2009. This article is published with open access at Springerlink.com

\begin{abstract}
Background Evidence-based surgery (EBS) is stressed to increase efficiency and health care quality, but not all surgeons and surgical nurses use EBS in clinical practice. To define future tailor-made interventions to improve evidence-based behavior, the aim of this study was to determine the attitude and awareness among surgeons and surgical nurses as to the paradigm of EBS and the barriers experienced when practicing EBS.

Methods In this cross-sectional study, surgeons and surgical nurses at a university hospital in Amsterdam were invited to complete the BARRIERS scale and McColl et al. questionnaire. An evidence quiz was composed for the surgeons.

Results Response rates were 67\% (29/43) for surgeons and $60 \%$ (73/122) for nurses. Attitudes toward EBS were positive. Among the surgeons, $90 \%$ were familiar with EBS terms, whereas only $40 \%$ of the nurses were. Common barriers for surgeons were conflicting results $(79 \%, 23 / 29)$ and the methodologic inadequacy of research reports (73\%, 21/29); and for nurses they were unawareness of EBS $(67 \%, 49 / 73)$ and unclear reported research $(59 \%, 43 / 73)$. Only about half of the convincing evidence presented in the quiz was actually applied.

Conclusions Surgeons have a positive attitude toward EBS and are familiar with EBS terminology, but conflicting
\end{abstract}

A. M. Knops $(\varangle) \cdot$ H. Vermeulen · D. T. Ubbink Department of Quality Assurance and Process Innovation, Academic Medical Center, University of Amsterdam, PO Box 22700, 1100 DE Amsterdam, The Netherlands

e-mail: a.m.knops@amc.nl

D. A. Legemate - D. T. Ubbink

Department of Surgery, Academic Medical Center, University of Amsterdam, PO Box 22700, 1100 DE Amsterdam, The Netherlands results and methodologic shortcomings of research reports are major barriers to practicing EBS. Continual confrontations with available evidence through frequent critical appraisal meetings or grand rounds and using more aggregate sources of evidence are advocated. Nurses can probably benefit from EBS training focusing on basic skills. Finally, collaboration is needed among surgeons and nurses with the same zest about EBS.

\section{Introduction}

During the last two decades the paradigm of evidencebased medicine (EBM) has been introduced and then fostered by societal and patients' demand for professional and resource accountability in health care [1]. Nowadays, executive boards stress the use of EBM to prevent unsafe practices that lack empirical support to reduce unacceptable individual variance and ultimately to increase efficiency and health care quality [2].

Reality, however, proves that not all health care professionals use EBM in their daily practice. In general medicine, roughly half of all medical treatments are evidence-based and only about one-fourth of all surgical treatments were found to be based on evidence [3,4]. This is related to a lack of surgical evidence, the fact that surgical questions cannot always be answered by a randomized clinical trial, and restrictive inclusion criteria that hamper application of trial recommendations to the average surgical patient [5]. Clinicians also report a lack of knowledge and basic skills-particularly about searching the literature and critical appraisal [6] — as major barriers to practicing EBM as well as a lack of personal time [7]. 
It often requires a joint venture between surgeons and surgical nurses to make evidence-based surgery (EBS) work. For example, evidence is accumulating that fasttrack surgery is useful for enhancimg patient recovery [8]. This result can only be achieved when surgeons and nurses collaborate. However, nurses also were found to face difficulties with EBS in that they believe research reports are too "academic" and do not offer the desired level of clinical direction [9].

Improvements in evidence-based behavior can only be realized, and measured, if awareness of EBS and a positive attitude toward EBS are secured first [10]. Moreover, it has been suggested that strategies to promote change in clinical practice are more likely to be successful if based on an analysis of barriers and facilitators specific to the context [11]. To our knowledge, awareness of EBS, attitude, and barriers to the utilization of research findings have not yet been investigated simultaneously among physicians and nurses in the surgical realm.

Therefore, the aim of this study was to determine the attitudes and awareness among surgeons and surgical nurses as to the principles of EBS and the barriers experienced when practicing EBS to be able to define future tailor-made interventions to improve evidence-based behavior in surgery.

\section{Methods}

\section{Context}

This study was carried out at the Department of Surgery of the Academic Medical Center (AMC), a university teaching hospital in Amsterdam, The Netherlands. It is part of a larger, ongoing inventory regarding attitudes, awareness, and perceived barriers among doctors and nurses of several specialties within the hospital. At the Department of Surgery, learning EBM is encouraged through yearly EBS courses and by producing critically appraised topics (CATs) based on actual routine clinical questions [12]. Although the surgical nurses are not yet involved in these local EBS initiatives, they are offered some courses to learn EBS skills.

To assess the extent to which the EBS principles are known and applied, a questionnaire and a quiz were developed. All surgeons, surgical trainees, and qualified nurses on all surgical wards were invited to complete the questionnaire.

\section{Questionnaire}

The questionnaire combined the BARRIERS scale and the McColl questionnaire [7, 13]. The BARRIERS scale investigates health care professionals' perceptions of barriers to the utilization of research findings in clinical practice. The scale has been validated and used in various settings worldwide [14-16]. The McColl et al. questionnaire addresses attitude, awareness, and actual use of EBM. It has also been applied ubiquitously [7, 17, 18]. In this study, "attitude" was defined as the mind-set of the responders as to the principles of EBM; "awareness" is defined as familiarity with the meaning of certain EBM terms.

Other validated instruments, such as the Berlin questionnaire and the Fresno test, objectively measure EBM skills [19, 20]. These measures were considered inappropriate in this study as their questionnaires aim to evaluate the effectiveness of an EBS course, whereas in our situation only optional EBS courses had been available.

Because the BARRIERS scale and the McColl et al. questionnaire are in English, they were translated into Dutch by the process of forward-backward translation [21]. Subsequently, a reliable Cronbach's $\alpha$ value of 0.95 was recalculated for the part containing the BARRIERS scale.

\section{EBS quiz}

Available CATs were used to measure if surgeons and trainees were aware of the surgical evidence communicated and how much of the evidence was in agreement with present practice. Two staff surgeons (D.A.L. and the head of the department) were consulted to select 20 CATs, representative for the four surgical subspecialties (general, gastroenterologic, trauma, and vascular surgery) and reflecting knowledge any member of the Department of Surgery should have. The EBS quiz consisted of multiplechoice questions formulated for each of the 20 topics. An example is shown in Table 1 , considering evidence that polyethylene glycol is not indicated to prepare the bowel before colon surgery [22] The other questions and corresponding anwers can be found in the Appendix).

During one of the surgical research meetings, the EBM quiz was conducted by showing each of the 20 questions for 30 seconds to allow the attendees to fill in their answer on a reply form. Correct answers were defined by the conclusion of the CAT and the actual policy of the surgical department based on the local guidelines and protocols available.

\section{Data analysis}

Means and standard deviations (SD) were calculated after checking for normal distribution. Student's $t$-test was used to compare the means of the attitude scores toward EBS between certain subgroups (surgeons and nurses, males and females, staff and nonstaff personnel). Correlation coefficients (according to Pearson's or Spearman's, as determined 
Table 1 Example of a question in the quiz

Question: Is bowel preparation with polyethylene glycol needed for elective colon and rectal surgery? (Considering anastomotic leakage, the wound, and intraabdominal infectious complications)

A: I am not aware of any evidence about this topic.

B: I am aware of the evidence about this topic: It was not conclusive (i.e., there are no results, or contradictory, results available).

C: I am aware of the evidence about this topic: We also apply this evidence (i.e., comparative research reports are available and show a difference in effect). Present practice in this hospital is in agreement with these research reports.

D: I am aware of the evidence about this topic, but we do not apply this evidence (i.e., comparative research reports are available and show a difference in effect, but present practice in this hospital is not in agreement with these research reports).

Correct answer is $\mathrm{C}[22]$

by the presence or absence of a normal distribution, respectively) and scatter graphs were made to find possible correlations between age or working experience in health care and attitudes toward EBS.

Nonparametric Kruskal-Wallis and Mann-Whitney tests were used for subgroup analysis. Statistical analysis was performed using SPSS 15.0 (SPSS, Chicago, IL, USA).

\section{Results}

We achieved response rates of $67 \%$ (29/43) for the surgeons and trainees and 60\% (73/122) for the nurses. Personal characteristics of the respondents were representative for the doctors and nurses working on the surgical wards (Table 2).

\section{McColl questionnaire}

Current attitudes of doctors and nurses toward EBS are shown in Table 3. Doctors in particular were extremely positive. They never disagreed with the statement: "practicing EBS improves patient care" (mean score 78, SD 14). Actual surgical practice was considered to be $45 \%$ (SD $16 \%$ ) evidence-based. Nurses' attitude toward the current promotion of EBS was welcoming, although there was wide variation in response (mean score 52, SD 21). Senior nurses appeared to have a significantly more positive attitude toward the principle of EBS than registered nurses $(P=$ $0.01)$ and considered research findings in daily practice significantly more useful $(P=0.005)$. When comparing surgeons to nurses, surgeons were found to have a more positive attitude toward EBS $(P=0.00)$, judged their colleagues to be more positive toward EBS $(P=0.00)$, and thought research findings were more useful in daily practice. No correlations between age, sex, or work experience in health care and attitude toward EBS were found.

Figure 1 shows the extent to which surgeons said they understand the most cited terms in articles about EBM that are likely to be relevant for surgical practice. Approximately $90 \%$ of the surgeons showed (some) understanding of six of eight terms. Particularly "absolute risk increase" and "confidence interval" were known by all surgeons. We added "fixed event rate" and "random benefit ratio"nonexisting dummy terms - as indicators of possible socially desirable answering [23]. Overall, the dummy terms scored a lower understanding than the other terms, as can be seen in Fig. 1 (approximately $40 \%$ compared to $90 \%$ of the existing terms). Hence, overestimation of competence was less likely in our study, which makes the scores about reported knowledge more reliable.

Table 2 Demographic data for respondents to the questionnaire

\begin{tabular}{lllll}
\hline Parameter & Responding surgeons (no.) & All surgeons (no.) & Responding nurses (no.) & All nurses (no.) \\
\hline Sex & & & & \\
$\quad$ Males & $18(62 \%)$ & $27(63 \%)$ & $16(22 \%)$ & $20(16 \%)$ \\
$\quad$ Females & $11(38 \%)$ & $16(37 \%)$ & $57(78 \%)$ & $102(84 \%)$ \\
Age (years), mean \pm SD & $38 \pm 8$ & $40 \pm 10$ & $37.6 \pm 13.0$ & $39.7 \pm 12.0$ \\
Experience & & & & $13(18 \%)$ \\
$\quad$ Trainees & $14(48 \%)$ & $20(47 \%)$ & $60(82 \%)$ & $15(12 \%)$ \\
$\quad$ Surgeons & $15(52 \%)$ & $23(54 \%)$ & 16 years (IQR 4-27)
\end{tabular}

a Experience for the nurses measures « Registered nurses » in place of « Trainees » and « Senior nurses » instead of « Surgeons » $I Q R$ interquartile range 
Table 3 Current attitudes toward experience-based medicine

\begin{tabular}{|c|c|c|}
\hline Attitudinal measures & $\begin{array}{l}\text { Surgeons' } \\
\text { score }\end{array}$ & $\begin{array}{l}\text { Nurses' } \\
\text { score }\end{array}$ \\
\hline Your current attitude toward EBS. Welcoming $\leftrightarrow$ Extremely welcoming & $76(10)^{\mathrm{a}}$ & $52(21)^{\mathrm{b}}$ \\
\hline Practicing improves patient care. Totally disagree $\leftrightarrow$ Totally agree & $78(14)$ & $72(18)$ \\
\hline What percentage of your clinical practice is evidence-based? $0 \% \leftrightarrow 100 \%$ & $45(16)$ & $45(22)$ \\
\hline Attitude of your colleagues toward EBS. Welcoming $\leftrightarrow$ Extremely welcoming & $67(13)^{\mathrm{c}}$ & $48(19)$ \\
\hline How useful are research findings in daily practice? Useless $\leftrightarrow$ Extremely useful & $69(15)^{\mathrm{d}}$ & $60(17)^{\mathrm{e}}$ \\
\hline EBS is of limited value in surgical practice because there is a lack of scientific base. Totally disagree $\leftrightarrow$ Totally agree & $44(18)$ & $50(18)$ \\
\hline $\begin{array}{l}\text { Implementing EBS, however worthwhile as an ideal, places another demand on already overloaded surgeons/nurses. } \\
\text { Totally disagree } \leftrightarrow \text { Totally agree }\end{array}$ & $56(21)$ & $53(22)$ \\
\hline
\end{tabular}

Scores can range from 0 to 100

Results are the means, with the standard deviation in parentheses

a Surgeons more positive than nurses $(P=0.00)$

b Senior nurses more positive than registered nurses $(P=0.01)$

${ }^{c}$ Surgeons more positive than nurses $(P=0.00)$

${ }^{\mathrm{d}}$ Surgeons more positive than nurses $(P=0.016)$

e Senior nurses more positive than registered nurses $(P=0.005)$

Fig. 1 Surgeons' and nurses' knowledge of evidence-based surgery (EBS) terms. The last two terms are meaningless "dummy terms." (1) I understand the meaning and could explain to others. (2) I partially understand the meaning. (3) I do not understand the meaning but would like to.

(4) I do not understand the meaning; it would be of no use to understand. (5) Missing value. $*$ N.N.H. number needed to harm; $* *$ N.N.T. number needed to treat

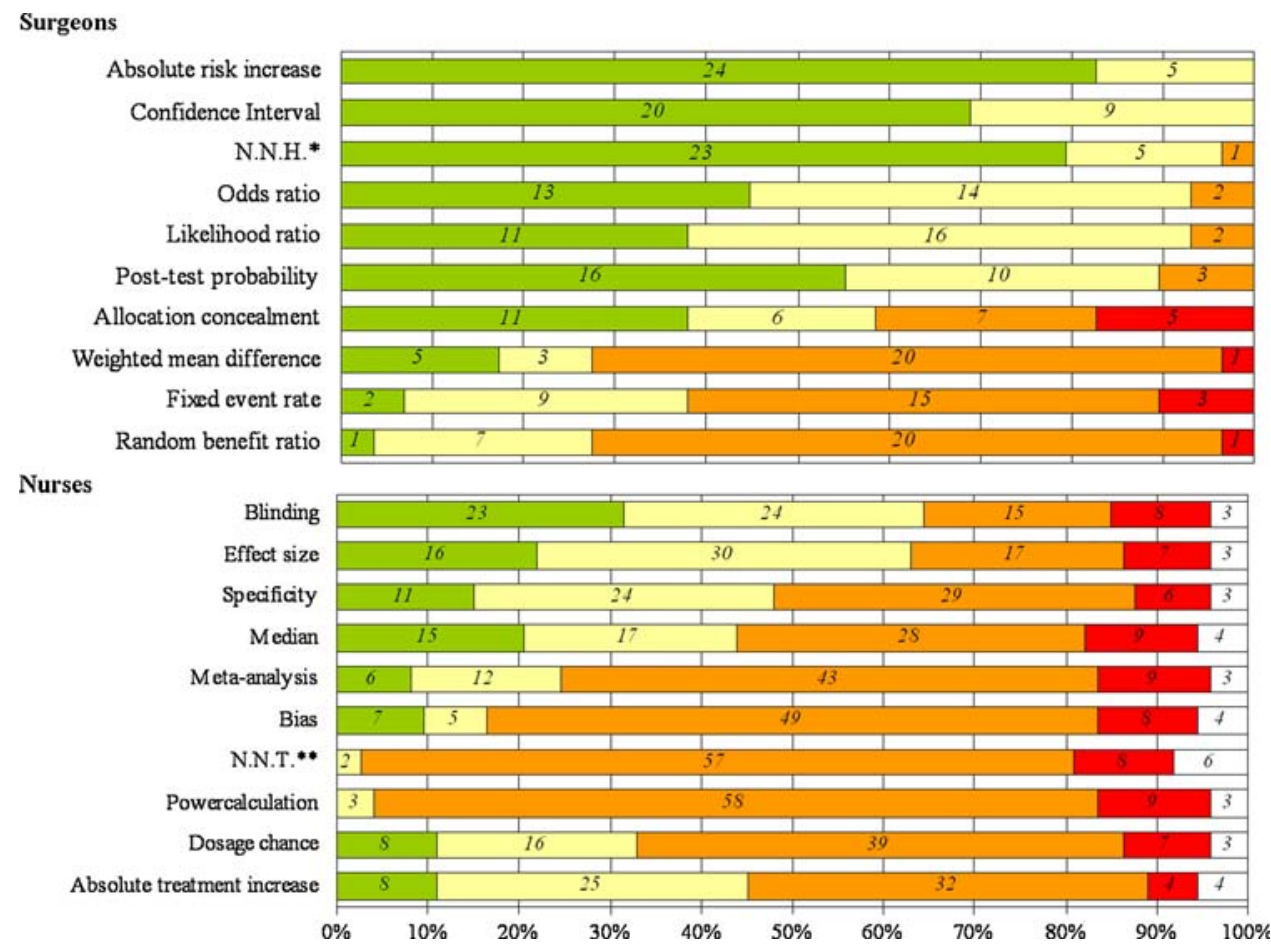

Ten common terms used in articles about EBS in the nurses' surgical practice were presented to the nurses. They were less aware of EBS terms than surgeons (about 40\%). "Absolute treatment increase" and "dosage chance" were inserted as dummy terms. Their understanding of these terms was similar to that of the existing terms (approximately $40 \%$ ), suggesting socially desirable answering to this part of the questionnaire.

\section{BARRIERS scale}

A five-point scale was used to rate the extent to which 29 statements were considered a barrier to using research in practice. By dichotomizing the answers, a list of major barriers for surgeons and nurses was produced (Table 4).

To surgeons, major barriers were the conflicting results $(79 \%, 23 / 29)$ and methodologic inadequacies $(73 \%, 21 / 29)$ 
Table 4 Barriers to research utilization

\begin{tabular}{|c|c|c|c|}
\hline $\begin{array}{l}\text { Five greatest barriers for surgeons }(n=29) \\
\text { and nurses }(n=73)\end{array}$ & $\begin{array}{l}\text { Moderate or } \\
\text { great barrier (no.) }\end{array}$ & $\begin{array}{l}\text { Small or } \\
\text { no barrier (no.) }\end{array}$ & No opinion (no.) \\
\hline \multicolumn{4}{|l|}{ Surgeons } \\
\hline Literature reports conflicting results & $23(79 \%)$ & $6(20 \%)$ & - \\
\hline Research has methodologic inadequacies & $21(73 \%)$ & $5(17 \%)$ & $3(10 \%)$ \\
\hline Insufficient time on the job to implement new ideas & $19(66 \%)$ & $10(35 \%)$ & - \\
\hline Implications for practice not made clear & $18(62 \%)$ & $11(38 \%)$ & - \\
\hline Do not have time to read research & $16(55 \%)$ & $13(45 \%)$ & - \\
\hline \multicolumn{4}{|l|}{ Nurses } \\
\hline Unaware of the research & $49(67 \%)$ & $16(22 \%)$ & $8(11 \%)$ \\
\hline Do not have time to read research & $49(67 \%)$ & $16(22 \%)$ & $8(11 \%)$ \\
\hline Insufficient time on the job to implement new ideas & $48(66 \%)$ & $18(25 \%)$ & $7(10 \%)$ \\
\hline Research not reported clearly and readably & $43(59 \%)$ & $13(18 \%)$ & $17(23 \%)$ \\
\hline Implications for practice not made clear & $42(58 \%)$ & $20(27 \%)$ & $11(15 \%)$ \\
\hline
\end{tabular}

of research reports, whereas nurses mentioned the unawareness of research reports in the first place $(67 \%, 49 /$ 73). A lack of time (to read research reports and to implement new ideas) and unclear implications for practice were rated as major barriers by both surgeons and nurses.

To facilitate the utilization of data from medical research, both surgeons and nurses stressed constant involvement and reminding each other to practice EBS. Nurses claimed to need time to learn the basic skills of EBS. Surgeons thought articles should be selected and discussed based on their relevance to surgical practice. Subsequently, both groups asked for clear, practical documents (e.g., a protocol or guideline) that would be easily obtainable through a periodical newsletter or an online database.

\section{EBS quiz}

The quiz was completed by $86 \%(36 / 42)$ of the attendees at the meeting (Table 5). Their mean age was 33 years (SD 9.4 years).

Surgeons $(P=0.001)$ and surgical trainees $(P<0.001)$ showed significantly more awareness of evidence than interns. This was expected because the latter group had just recently joined the department and had received no previous EBM training.

Table 5 Respondents' answers to the quiz

\begin{tabular}{lll}
\hline Respondents & $\begin{array}{l}\text { Awareness of } \\
\text { evidence }(\%)\end{array}$ & $\begin{array}{l}\text { Application of } \\
\text { evidence }(\%)\end{array}$ \\
\hline Surgeons $(n=11)$ & 67 & 50 \\
Trainees $(n=12)$ & 67 & 41 \\
Interns $(n=10)$ & 26 & 19 \\
Researchers $(n=3)$ & 50 & 17 \\
\hline
\end{tabular}

Of the 20 questions, 8 referred to convincing evidence that was actually applied in surgical practice according to local guidelines and protocols. Surgeons $(n=11)$ answered affirmatively to these questions correctly in 50\% (44/88) and trainees $(n=12)$ in $41 \%(39 / 96)$, respectively. Thus, surgeons and trainees used nearly half of the convincing evidence as presented in the quiz, despite moderate to good awareness of the evidence itself.

\section{Discussion}

In this study, we have shown that surgeons at a university hospital in The Netherlands appear to have a positive attitude toward the paradigm of EBS and are familiar with EBS terminology but that conflicting results and methodologic shortcomings of research reports appear to be major barriers to practicing EBS. These barriers have also been reported in nonsurgical settings [24-26]. To implement EBM successfully in daily practice, further teaching of the EBM principles to nurses and stimulating and reminding surgical colleagues to apply these principles is required.

Although not specified by the surgeons stating this barrier, the "major methodologic shortcomings" generally are well known flaws in the design or conduct of the studies that jeopardize study validity and thus the strength of the evidence. Among the shortcomings are nonrandomized trials (most surgical studies are case series), no allocation concealment or blinding used (patients and surgeons are usually aware of the treatment given), insufficient follow-up, no intention-to-treat principle applied, and small sample size [27].

Surprisingly, surgeons and trainees indicated that they use only about half of the convincing evidence as presented during the quiz, even though it was decided earlier in the group of surgeons to include this evidence in local guidelines or to change local surgical policy. Apparently, the 
decision whether to apply evidence is often difficult (e.g., because of other available evidence showing a favorable outcome on one endpoint but a harmful effect on another relevant clinical endpoint).

Nurses had a moderately positive attitude toward the principles of EBS but were rather unfamiliar with EBS sources and terminology. They indicated that unawareness of research reports was the major barrier to using research findings in surgical nursing practice.

To facilitate the practice of EBS, both surgeons and nurses suggested constant involvement of EBS in daily practice, interactive education, and the availability of a digital expert system to support practice, which is also described in the literature [11,28].

A limitation of this study design is that self-perception of attitude, awareness, and barriers toward EBS were assessed. One would rather determine these parameters during "realtime" surgical practice and ultimately measure if patient outcomes improve owing to the application of surgical evidence by surgeons and nurses. Although the number of instruments to evaluate EBS is growing [29], valid assessment instruments to evaluate EBS behavior are still lacking.

Second, the absolute number of participants in this study was limited and derived from one university hospital only. However, the response rate of surgeons and nurses within our department was satisfactory. Our findings may be indicative of the situation in other (academic) centers because similar implementation barriers and positive attitudes regarding EBM have been reported [7, 14, 26]. Despite these similarities, further research is needed in other specialties and other medical centers to see if general recommendations can be made on interventions to promote EBM.
Implementation of EBS needs further improvement. Several tailor-made interventions have become clear from our findings and experience in creating a professional environment that incorporates EBS. First, continual confrontation with available evidence through frequent critical appraisal meetings or grand rounds is necessary [30]. Second, as our nurses were unfamiliar with EBS and received less postgraduate EBS training than surgeons, it is expected that they will benefit from EBS training focusing on basic skills, integrated in everyday surgical nursing practice $[31,32]$. Third, surgeons may enhance their efficiency by using more aggregate sources of evidence [33], such as the National Guideline Clearinghouse or BMJ Clinical Evidence. Finally, collaboration is advocated among doctors and nurses with the same zest for EBS.

Acknowledgments We are grateful to Dr. K. Boer, at the Department of Clinical Epidemiology, Biostatistics, and Bioinformatics at the time of the study, who helped with the forward-backward translation of the questionnaires. We also thank Prof. Dr. D.J. Gouma of the Surgery Department for facilitating the survey and for his help selecting the CATs.

Open Access This article is distributed under the terms of the Creative Commons Attribution Noncommercial License which permits any noncommercial use, distribution, and reproduction in any medium, provided the original author(s) and source are credited.

\section{Appendix}

See Table 6

Table 6 Critically appraised topics on which the EBS quiz was based

\begin{tabular}{|c|c|c|}
\hline $\begin{array}{l}\text { Question } \\
\text { no. }\end{array}$ & Critically appraised topic & $\begin{array}{l}\text { Correct } \\
\text { answer }\end{array}$ \\
\hline 1 & Can posttraumatic dystrophy be prevented by supplementing vitamin C? & $\mathrm{C}$ \\
\hline 2 & PTFE or vein grafts for supragenual femoropopliteal bypass surgery? (Regarding primary and secondary patency) & $\mathrm{C}$ \\
\hline 3 & $\begin{array}{l}\text { Preoperative or postoperative chemoradiation therapy for operable stage II/III rectal carcinomas? (Considering 5-year } \\
\text { survival) }\end{array}$ & B \\
\hline 4 & $\begin{array}{l}\text { Does laparoscopic appendectomy have diagnostic and therapeutic advantages compared to open appendectomy? } \\
\text { (Considering duration of surgery, complications, time to recovery, and preventing a negative appendectomy) }\end{array}$ & $\mathrm{C}$ \\
\hline 5 & $\begin{array}{l}\text { Does additional, early MRI have additional value for the management of a clinically suspected scaphoid fracture with a } \\
\text { normal radiograph? }\end{array}$ & $\mathrm{C}$ \\
\hline 6 & $\begin{array}{l}\text { Is it better to perform carotid desobstruction under local anesthesia than under general anesthesia? (Considering mortality } \\
\text { and morbidity) }\end{array}$ & B \\
\hline 7 & Should the colorectal anastomosis of a rectosigmoid resection be tested peroperatively? (To prevent anastomotic leakage) & $\mathrm{C}$ \\
\hline 8 & Is a COX-2 antagonist a better postoperative analgesic than diclofenac? & B \\
\hline 9 & $\begin{array}{l}\text { Should a subcapital humerus fracture be treated conservatively or operatively? (Considering shoulder function, pain, and } \\
\text { time to recovery) }\end{array}$ & B \\
\hline 10 & $\begin{array}{l}\text { Should donor nephrectomy in healthy, living volunteers be performed via a laparoscopic or open procedure? (Considering } \\
\text { donor safety, graft safety, preoperative incidents, reoperations, and complications) }\end{array}$ & $\mathrm{D}$ \\
\hline
\end{tabular}


Table 6 continued

Question Critically appraised topic Correct

no. $\quad$ answer

11 Is bowel preparation with polyethylene glycol useful for elective colon surgery or rectal surgery? (Regarding complications C of the wound, complications of the anastomosis, and intraabdominal infections)

12 Should intensive-care patients to be given either prepyloric or postpyloric drip-feeding to prevent aspiration pneumonia? B

13 Is it useful to provide long-term thrombosis prophylaxis (19-23 days) with anti-Xa after hip fracture surgery to prevent D deep venous thrombosis and pulmonary embolism? (Considering major bleeding)

14 Is it better to either perform stripping or Closure procedure (radiofrequency obliteration) of the greater saphenous vein? $\mathrm{C}$

(Regarding quality of life, complications, and time to recovery)

15 Should omentum-plasty be applied to prevent anastomotic leakage for colorectal anastomoses? B

16 Which additional test (CT or ultrasonography) is most useful to diagnose acute appendicitis in young, fertile women? D

17 Should percutaneous screw fixation be applied for disabling pain after an isolated pubic fracture? B

18 Is drug treatment of an abdominal aortic aneurysm useful to prevent its growth? B

19 Is it useful to perform abdominal drainage in patients with chronic liver infections (hepatitis/cirrhosis) after partial liver $\mathrm{C}$ resections?

\section{References}

1. Claridge JA, Fabian TC (2005) History and development of evidence-based medicine. World J Surg 29:547-553

2. Donald A, Greenhalgh T (2000) A hands-on guide to evidence based health care: Practice and Implementation. Oxford, Blackwell Science

3. Ellis J, Mulligan I, Rowe J et al (1995) Inpatient general medicine is evidence based: a-team, Nuffield department of clinical medicine. Lancet 346:407-410

4. Howes N, Chagla L, Thorpe M et al (1997) Surgical practice is evidence based. Br J Surg 84:1220-1223

5. Slim K (2005) Limits of evidence-based surgery. World J Surg 29:606-609

6. McAlister FA, Graham I, Karr GW et al (1999) Evidence-based medicine and the practicing clinician. J Gen Intern Med 14: 236-242

7. McColl A, Smith H, White P et al (1998) General practitioner's perceptions of the route to evidence based medicine: a questionnaire survey. BMJ 316:361-365

8. Kehlet H, Wilmore DW (2008) Evidence-based surgical care and the evolution of fast-track surgery. Ann Surg 248:189-198

9. McCaughan D, Thompson C, Cullum N et al (2002) Acute care nurses' perceptions of barriers to using research information in clinical decision-making. J Adv Nurs 39:46-60

10. Cabana MD, Rand CS, Powe NR et al (1999) Why don't physicians follow clinical practice guidelines? A framework for improvement. JAMA 282:1458-1465

11. Grol R, Grimshaw J (1999) Evidence-based implementation of evidence-based medicine. Jt Comm J Qual Improv 25:503-513

12. Ubbink DT, Legemate DA (2004) Evidence-based surgery. Br J Surg 91:1091-1092

13. Funk SG, Champagne MT, Wiese RA et al (1991) BARRIERS: the barriers to research utilization scale. Appl Nurs Res 4:39-45

14. Bryar RM, Closs SJ, Baum G et al (2003) The Yorkshire BARRIERS project: diagnostic analysis of barriers to research utilisation. Int J Nurs Stud 40:73-84

15. Hutchinson AM, Johnston L (2004) Bridging the divide: a survey of nurses' opinions regarding barriers to, and facilitators of, research utilization in the practice setting. J Clin Nurs 13:304-315

16. Parahoo K, McCaughan EM (2001) Research utilization among medical and surgical nurses: a comparison of their self reports and perceptions of barriers and facilitators. J Nurs Manag 9: $21-30$

17. Toulkidis V, Donnelly NJ, Ward JE (2005) Engaging Australian physicians in evidence-based medicine: a representative national survey. Intern Med J 35:9-17

18. Veness M, Rikard-Bell G, Ward J (2003) Views of Australian and New Zealand radiation oncologists and registrars about evidencebased medicine and their access to Internet based sources of evidence. Australas Radiol 47:409-415

19. Fritsche L, Greenhalgh T, Falck-Ytter Y et al (2002) Do short courses in evidence based medicine improve knowledge and skills? Validation of Berlin questionnaire and before and after study of courses in evidence based medicine. BMJ 325: 1338-1341

20. Ramos KD, Schafer S, Tracz SM (2003) Validation of the Fresno test of competence in evidence based medicine. BMJ 326: 319-321

21. Brislin RW (1986) The wording and translation of research instruments. In: Field methods in cross-cultural research. Sage, Beverly Hills, CA, pp 137-164

22. Slim K, Vicaut E, Panis Y et al (2004) Meta-analysis of randomized clinical trials of colorectal surgery with or without mechanical bowel preparation. Br J Surg 91:1125-1130

23. Oliveri RS, Gluud C, Wille-Jorgensen PA (2004) Hospital doctors' self-rated skills in and use of evidence-based medicine: a questionnaire survey. J Eval Clin Pract 10:219-226

24. Beresford EB (1991) Uncertainty and the shaping of medical decisions. Hastings Cent Rep 21:6-11

25. Grol R (2001) Improving the quality of medical care: building bridges among professional pride, payer profit, and patient satisfaction. JAMA 286:2578-2585

26. Sur RL, Scales CD Jr, Preminger GM et al (2006) Evidencebased medicine: a survey of American Urological Association members. J Urol 176:1127-1134

27. McLeod RS (1999) Issues in surgical randomized controlled trials. World J Surg 23:1210-1214

28. Grol R, Grimshaw J (2003) From best evidence to best practice: effective implementation of change in patients' care. Lancet 362 : $1225-1230$

29. Shaneyfelt T, Baum KD, Bell D et al (2006) Instruments for evaluating education in evidence-based practice: a systematic review. JAMA 296:1116-1127 
30. Urschel JD (2005) How to analyze an article. World J Surg 29:557-560

31. Coomarasamy A, Khan KS (2004) What is the evidence that postgraduate teaching in evidence based medicine changes anything? A systematic review. BMJ 329:1017
32. Parkes J, Hyde C, Deeks J et al (2001) Teaching critical appraisal skills in health care settings. Cochrane Database Syst Rev 3: CD001270

33. Sigouin C, Jadad AR (2002) Awareness of sources of peerreviewed research evidence on the Internet. JAMA 287:2867-2869 